\title{
Identification of Positive and Negative Regulatory Regions Controlling Expression of the Cartilage Matrix Protein Gene
}

\author{
IBOLYA KISS, ${ }^{1 *}$ ZSUZSA BŐSZE,${ }^{2} \dagger$ PIROSKA SZABÓ, ${ }^{1}$ RENCENDORJ ALTANCHIMEG,${ }^{1} \ddagger$ \\ ENDRE BARTA, ${ }^{1}{ }^{\dagger}$ AND FERENC DEÁK ${ }^{1}$ \\ Institute of Biochemistry ${ }^{1}$ and Institute of Genetics, ${ }^{2}$ Biological Research Center of the \\ Hungarian Academy of Sciences, P.O. Box 521, H-6701 Szeged, Hungary
}

Received 16 November 1989/Accepted 1 February 1990

\begin{abstract}
A complex pattern of regulation of the cartilage matrix protein gene was revealed by transient expression experiments. A minimal promoter from positions -15 to +64 functioned in chondrocytes and fibroblasts. An enhancer located in the first intron exerted chondrocyte-specific stimulation on the minimal promoter activity. The same fragment, however, had a negative effect in fibroblasts. Between -334 and -15 , a silencer was found which inhibited the gene expression driven from its homologous as well as heterologous promoters both in chondrocytes and fibroblasts. Additional positive and negative control regions were mapped further upstream of the promoter.
\end{abstract}

Cartilage plays an important role in vertebrate development. Chondrocytes, the cellular components of cartilage, secrete a characteristic set of macromolecules (11). Genes for cartilage proteins are activated in a sequential order concomitant with chondrogenesis $(6,18,24)$, indicating the role of both common and distinct regulatory elements in their control. Data are available concerning only the regulation of the type II collagen gene $(12,19)$. In an attempt to investigate the molecular events underlying the cartilagespecific gene expression, we isolated genes for chicken link protein (17) and cartilage matrix protein (CMP) (16).

CMP is a major noncollagenous glycoprotein of hyalin cartilage that forms oligomers via disulfide bridges and may have a function in matrix assembly $(1,22)$. The monomer of chicken CMP has a molecular weight of 54,000 . The protein consists of two repeated I domains connected by an EGF module, indicating a common evolutionary origin with various protein families $(1,15,16,23)$.

We have located recently the putative promoter of the CMP gene (16). To identify cis-acting regulatory regions, selected fragments from the $5^{\prime}$ end and the first intron of the gene were coupled to the chloramphenicol acetyltransferase (CAT) reporter gene driven from homologous as well as heterologous promoters. In the names of the constructs, + and - indicate whether the fragment was inserted in the direct or opposite orientation, respectively, relative to the CAT gene. All positions are given in base pairs from the first $\mathrm{T}$ of the TATA motif of the CMP gene. Primary chondrocytes were obtained from sterna of 14-day-old chicken embryos. Parallel plates of chondrocyte and fibroblast cultures were transfected with the recombinant clones by the calcium phosphate method (10) and assayed for CAT activity (9). To correct for the transfection efficiency, parallel plates were also transfected with a control plasmid. The results are expressed as a percentage of the value of CAT activity obtained with the control plasmid (relative CAT activity).

Far-upstream negative regulatory region. The 5 '-flanking

\footnotetext{
* Corresponding author.

$\dagger$ Present address: Agricultural Biotechnology Center, H-2101 Gödöllő, Hungary.

$\ddagger$ Present address: Institute of Biotechnology, Academy of Sciences, Ulan Bator 51, Mongolia.
}

fragments of the CMP gene were inserted into the HindIII site of pTK - CAT (21). From -10.7 to -2.4 kilobases $(\mathrm{kb})$, no enhancer which was able to increase the expression of the CAT gene driven from the heterologous thymidine kinase (TK) promoter was found (Table 1). Insertion of $\mathrm{H}_{A}$ and $\mathrm{H}_{B}$ fragments, however, decreased the promoter activity in chondrocytes by 10- and 5-fold, respectively, in either orientation. The observation that only a moderate inhibition was detected in fibroblasts indicates the presence of chondrocyte-specific negative regulatory elements from 6.8 to $10.7 \mathrm{~kb}$ upstream of the CMP gene.

Enhancer activity in the first intron. When the $1.2-\mathrm{kb}$ $B g l I I-N c o I$ fragment carrying the CMP promoter was fused to the CAT reporter gene (Fig. 1), very low activity was monitored upon transfection of chondrocytes with the parental plasmid pCMP . CAT - N (Fig. 2). The CAT synthesis decreased even further when fragment $B$ was deleted [pCMP . CAT - N $(-\mathrm{B})]$. The reason for the weak CMP promoter activity could be either the lack of a tissue-specific enhancer or the presence of a negative regulatory element in the 5 '-flanking region of the gene.

In an attempt to find a transcriptional enhancer, we inserted $\mathrm{P}_{\mathrm{a}}, \mathrm{P}_{\mathrm{b}}, \mathrm{P}_{\mathrm{c}}$, and additional overlapping fragments from the first intron into the CMP promoter-CAT constructs $5^{\prime}$ of the promoter. The effect of the $P_{c}$ fragment (+966 to about +3000 ) was rather inhibitory. Among the fragments tested, inclusion of the $P_{b}$ fragment $(+578$ to +966$)$ into pCMP . CAT - N(-B) resulted in the highest activity, corresponding to 10 - to 12 -fold stimulation in chondrocytes (Fig. 2). The enhancement was specific for chondrocytes, resulting in only a moderate increase in fibroblasts. The fact that the fragment was able to exert significant stimulation on the promoter activity in both orientations and in a configuration different from the natural one indicates the presence of enhancerlike elements between +578 and +966 . Furthermore, sequence analysis revealed the presence of an enhancer core (14) sequence motif in the fragment.

Deletion analysis. Insertion of the Rous sarcoma virus enhancer (20) into the SalI site of the parental plasmid in either orientation was not able to stimulate the CMP promoter activity more than twofold (data not shown), suggesting that a negative regulatory element resides in the $1.2-\mathrm{kb}$ BglII-NcoI fragment. 
TABLE 1. Modulation of CAT activity by 5 '-flanking HindIII fragments of the CMP gene when transcribed from the heterologous TK promoter

\begin{tabular}{lclll}
\hline \multirow{2}{*}{$\begin{array}{l}\text { Frag- } \\
\text { ment }\end{array}$} & Position & & \multirow{2}{c}{$\begin{array}{c}\text { Orientation in } \\
\text { pTK } \cdot C A T\end{array}$} & \multicolumn{2}{c}{$\begin{array}{c}\text { Relative CAT activity } \\
(\% \pm S D)^{b} \text { in: }\end{array}$} \\
\cline { 4 - 5 } & & & Chondrocytes & Fibroblasts \\
\hline $\mathrm{H}_{\mathrm{A}}$ & -10.7 to -8.0 & Direct & $13.7 \pm 0.8$ & $64.9 \pm 14.7$ \\
& & Opposite & $11.8 \pm 4$ & $46.4 \pm 24.7$ \\
$\mathrm{H}_{\mathrm{B}}$ & -8.0 to -6.8 & Direct & $14.3 \pm 5.1$ & $62.8 \pm 9.7$ \\
& & Opposite & $21.7 \pm 5.4$ & $42.2 \pm 9.7$ \\
$\mathrm{H}_{\mathrm{C}}$ & -6.8 to -5.8 & Direct & $76.8 \pm 18.9$ & $102 \pm 11.6$ \\
$\mathrm{H}_{\mathrm{D}}$ & -5.8 to -2.4 & Direct & $74.5 \pm 22.9$ & $56.4 \pm 0.7$ \\
\hline
\end{tabular}

${ }^{a}$ In kilobases from the TATA box of the CMP gene.

${ }^{b}$ Each value represents the mean from three to six independent transient transfection experiments and is expressed relative to the activity of contro plasmid pTK - CAT (100\%), which corresponds to 30 to $50 \%$ chloramphenicol acetylation.

To locate the negative regulatory region, fragments $\mathrm{A}, \mathrm{B}$, and $C$ spanning the 5 -flanking region of the CMP gene from positions -1136 to $-942,-942$ to -334 , and -334 to -15 , respectively, were removed in different combinations from the parental plasmid (Fig. 3). Figure 4 summarizes the effects of the deletions on gene expression in the absence and presence of the enhancerlike $P_{b}$ fragment. Removal of all three fragments, resulting in pCMP . CAT.$N(-A B C)$, in- creased the level of expression compared with that of the parental plasmid. Therefore, we concluded that sequences between -15 and +64 were sufficient to function as a minimal promoter.

In chondrocytes, deletion of fragment $\mathrm{C}$ yielded an eightfold increase in CAT activity [compare pCMP . CAT $\cdot \mathrm{N}(-\mathrm{C})$ with the parental plasmid]. Even constructs carrying the minimal promoter showed 3- to 12-fold higher levels of CAT synthesis than either of the constructs containing fragment $\mathrm{C}$. The data indicate, therefore, that fragment $\mathrm{C}$ harbors a strong negative regulatory element(s).

The presence of fragments A and B stimulated the minimal promoter activity about 2.5 -fold in chondrocytes [compare pCMP $\cdot$ CAT $\cdot N(-C)$ with pCMP $\cdot$ CAT $\cdot N(-A B C)$ in Fig. 4]. It demonstrates that an upstream positive control region located between -1136 and -334 contributes to the CMP promoter activity.

The intronic $P_{b}$ fragment, referred to as enhancer in Fig. 3 and 4, was introduced $5^{\prime}$ of the CMP promoter into the members of the deletion series. The construct harboring the enhancer fragment and the minimal promoter gave rise to the highest level of gene expression among the CMP-CAT derivatives in chondrocytes (Fig. 4). The promoter activity increased up to about $24 \%$ of that measured for pSV2 . CAT, which carries the strong simian virus 40 enhancer. This finding shows that the stimulation achieved by sequences

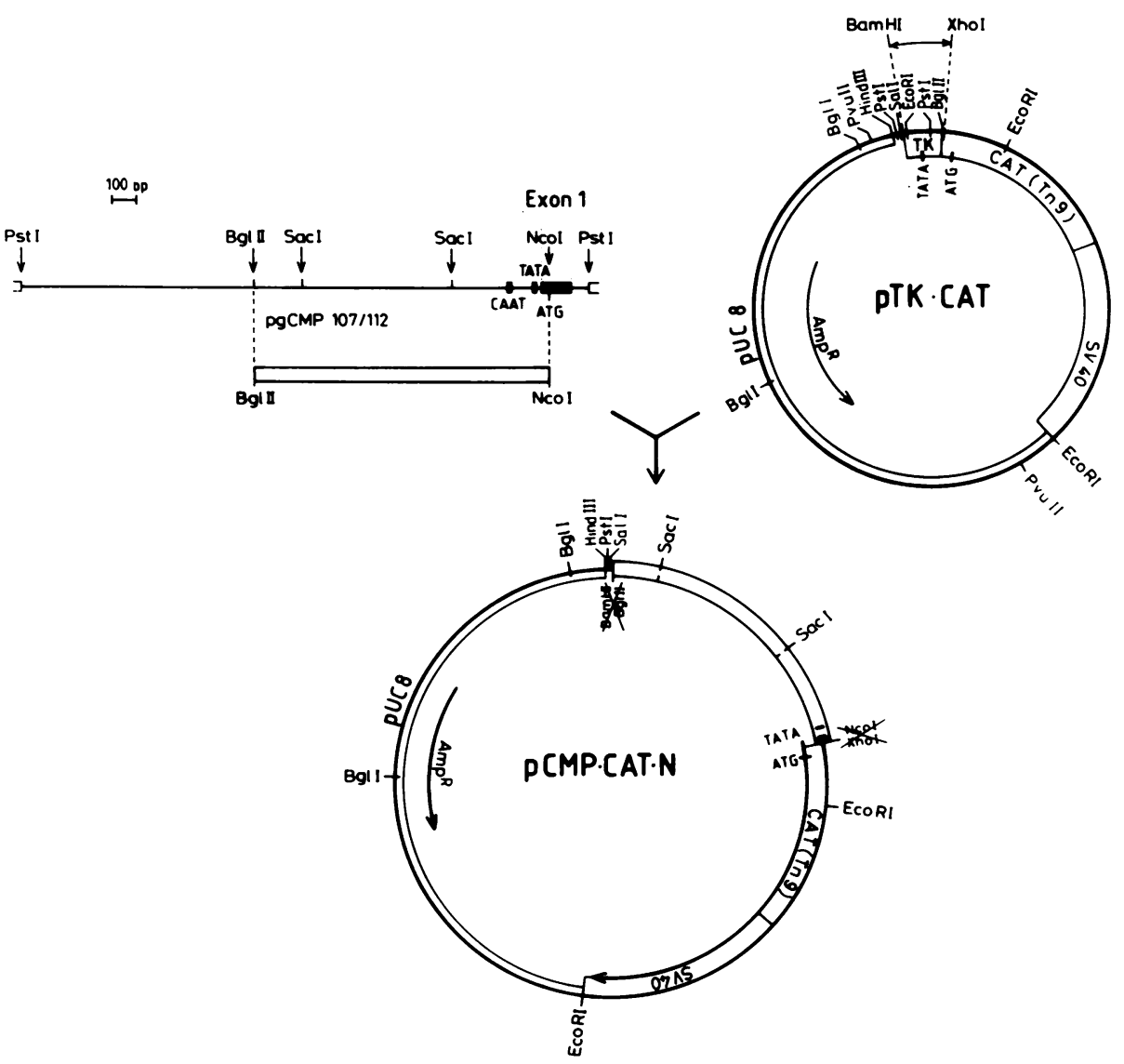

FIG. 1. Construction of the CMP promoter-CAT plasmid. The BamHI-XhoI fragment of pTK - CAT (21) carrying the TK promoter was replaced by the 1.2-kb BglII-NcoI fragment of subclone pgCMP107/112 after nuclease S1 treatment of the NcoI and XhoI ends. Relevant restriction sites are indicated. Sequence analysis revealed that pCMP . CAT - N carried the CMP promoter region from positions -1136 to +64 base pairs (bp). SV40, Simian virus 40. 


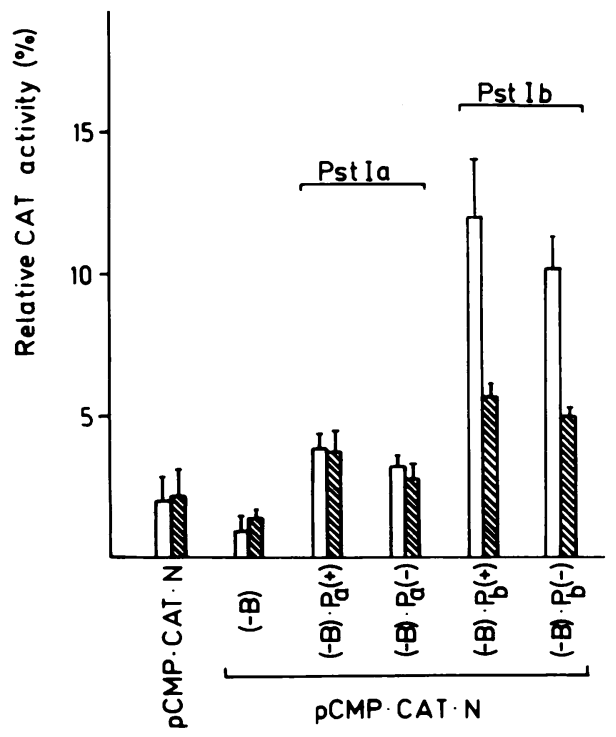

FIG. 2. Modulation of CAT activity by intronic fragments. Construct pCMP . CAT - N(-B) was generated by deleting the Sacl fragment ( -942 to -334 , fragment $B$ in Fig. 3) from the parental plasmid. $P_{a}(+224$ to +557$)$ and $P_{b}(+578$ to +966$)$ fragments were inserted in both orientations into the Pst I site of this plasmid. Cultures of $7.5 \times 10^{6}$ to $1 \times 10^{7}$ chondrocytes or $5 \times 10^{6}$ to $7 \times 10^{6}$ fibroblasts were transfected with $10 \mu \mathrm{g}$ of DNA 3 to $4 \mathrm{~h}$ after plating. CAT activity values obtained for each DNA from duplicate or triplicate plates were averaged. Relative CAT activity was calculated after the activity of the pSV2 . CAT (9) control was defined as $100 \%$. The histogram represents the averages \pm standard deviation determined from seven and five independent transfection experiments in chondrocytes $(\square)$ and fibroblasts $(\mathbb{Q})$, respectively.

located in the intronic $P_{b}$ fragment is within the range of effects of the viral enhancers.

The enhancer element also increased the level of gene expression when fragment $B$ was removed from the parental plasmid [Fig. 4, derivative $(-B)$ ], but in the presence of fragment $B$, it was rather inhibitory. This suggests further interactions between the intronic and upstream regulatory elements.

Cell-specific expression. Chicken embryo fibroblasts and

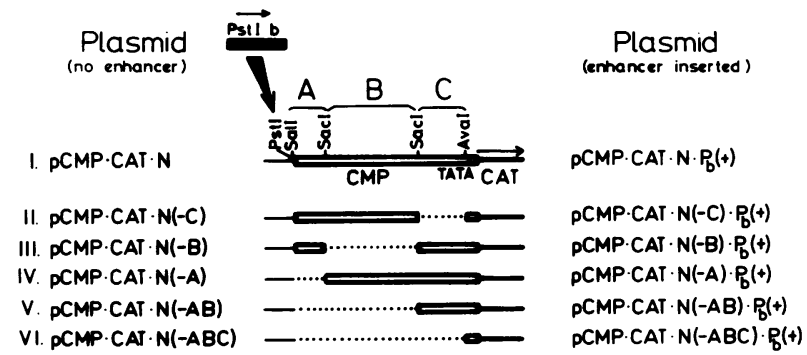

FIG. 3. Structure of CMP promoter-CAT plasmids containing deletions in the 5 '-flanking control region. Relevant restriction sites utilized for construction of the plasmids are indicated. SalI and AvaI ends were ligated directly, while T4 DNA polymerase treatment was used before joining SacI and AvaI as well as $S a l I$ and $S a c I$ ends to each other. The sizes and positions of deletions are represented by. dotted lines. The box labeled PstI $b$ corresponds to the intronic $P_{b}$ fragment, which carries enhancerlike activity. This fragment was inserted into one set of the constructs at the PstI site. rat hepatoma cells were also transfected with the constructs. It is interesting to note that the CMP minimal promoter was about fourfold more active in fibroblasts than in chondrocytes (Fig. 4). pCMP . CAT - N(-C) displayed the highest level of CAT synthesis in fibroblasts. The 26 -fold increase in activity upon removal of fragment $\mathrm{C}$ from the parental plasmid indicates that the negative control elements located in fragment $\mathrm{C}$ can inhibit the CMP promoter activity even more drastically in fibroblasts than in chondrocytes.

Each of the constructs harboring the enhancer fragment yielded a low level of CAT activity in fibroblasts, and only a slight increase was observed upon insertion of the $P_{b}$ fragment into pCMP . CAT - N(-B). To sum up, the $P_{b}$ fragment conferred positive regulation to the minimal promoter in chondrocytes, while in fibroblasts it repressed the gene expression more than threefold.

None of the constructs depicted in Fig. 3 was active in the Reuber-H56 rat hepatoma cell line $(5,25)$, giving relative CAT activity values below $2 \%$ in several independent experiments (data not shown).

Our data demonstrate that both the CMP promoter and the enhancer work in a tissue-specific manner. The minimal promoter is active in other connective tissue cells, such as fibroblasts, but not in cells of different origins. The enhancer fragment, however, functions only in chondrocytes and plays an important role in conferring chondrocyte-specific expression to the CMP gene. It may carry binding sites for both positive and negative factors, and the net effect depends on the actual concentrations of the different factors in a given tissue. The close proximity of positive and negative regulatory regions was reported for other genes $(7,13,26)$.

Fragment $\mathbf{C}$ acts as a silencer. To test whether the negative element located immediately upstream of the CMP promoter is capable of reducing the activity of heterologous promoters, we inserted fragment C into pTK . CAT at various positions both $5^{\prime}$ and $3^{\prime}$ of the reporter gene. Each construct displayed a markedly repressed CAT synthesis in either orientation compared with that of pTK . CAT, indicating that the fragment exerted a negative regulation on the TK promoter at all positions tested (Fig. 5). Therefore, we concluded that the fragment carries a silencer.

The levels of inhibition varied in the given constructs. When inserted in the opposite orientation, fragment $\mathrm{C}$ repressed the TK promoter activity by factors of 10 to 65 . Upon insertion in the direct orientation, the reduction was 33 -fold at position +1783 and less pronounced (3-fold) at positions -223 and -326 [compare derivative Pv2(+) with $\mathrm{P} 19(+)$ and Pv1(+) in Fig. 5]. Further studies are necessary to understand the molecular basis of differences in the silencer activity observed when placed $5^{\prime}$ of the promoter. Either the distance from the TK promoter or the nucleotide sequences surrounding the site of insertion may influence the silencing effect via protein-mediated interactions [compare derivative $\mathrm{B} 9(+)$ with $\mathrm{P} 19(+)$ and $\mathrm{Pv1}(+)$ in Fig. 5]. Nuclease $\mathrm{S} 1$ analysis confirmed that insertion of fragment $\mathrm{C}$ did not change the initiation site of transcription and that inhibition occurred at the level of transcription (data not shown). Negative regulation was not restricted to chondrocytes. Similar repression was observed in fibroblasts (data not shown).

Fragment C was also inserted into pRSV - CAT (8) at the SphI site located between the Rous sarcoma virus enhancer and promoter. Compared with that measured in pRSV . CAT, the relative CAT activity values measured in chondrocytes in the direct and opposite orientations dropped down to $38.7 \% \pm 6.7 \%$ and $19.3 \% \pm 6.1 \%$, respectively. 


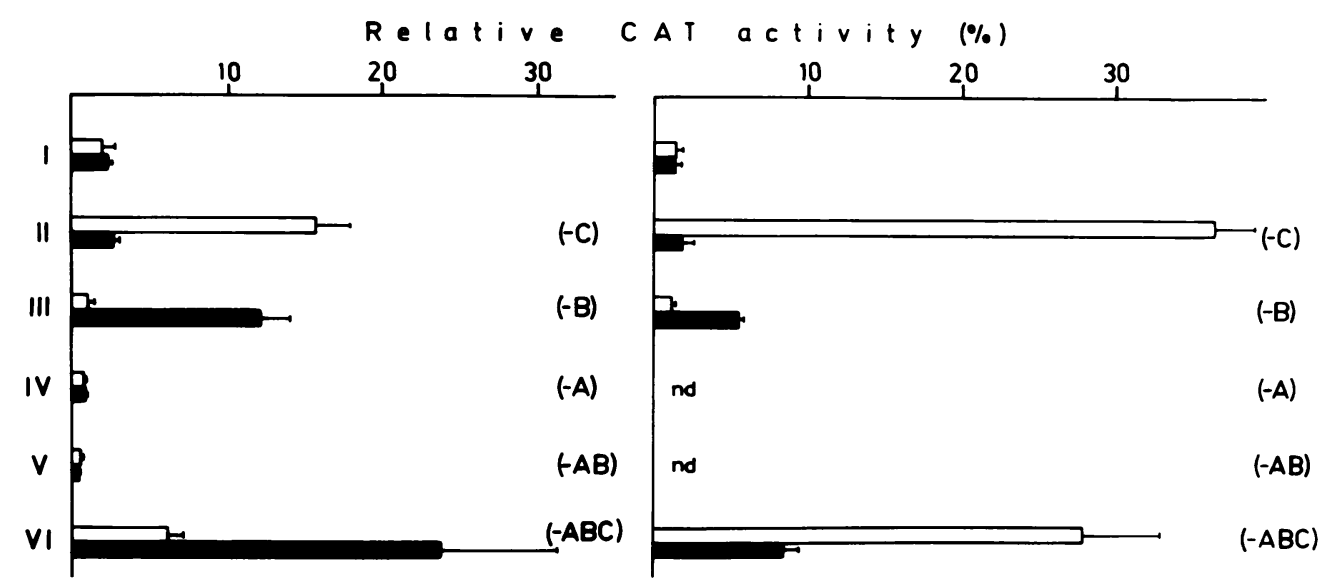

FIG. 4. Effect of removal of 5 '-flanking sequences on CMP promoter activity. Roman numerals designate the constructs as in Fig. 3, and the fragments deleted are indicated to the right of each graph. The histogram summarizes the results of four to nine independent transfection experiments. The relative CAT activity is given as percentage of the pSV2 . CAT control. Symbols: $\square$ and $\square$, with and without the enhancer (the intronic $P_{b}$ fragment of the CMP gene), respectively. nd, Not determined.

This observation demonstrates that the silencer is able to interfere with the Rous sarcoma virus enhancer and that the repression is also more pronounced in the opposite than in the direct orientation.

The results reported here identify several blocks of control elements dispersed at the $5^{\prime}$ end and in the first intron of the chicken CMP gene which modulate the activity of the minimal promoter. Stimulation was achieved by a region from -1136 to -334 and by a cartilage-specific enhancer residing between +578 and +966 . The presence of an

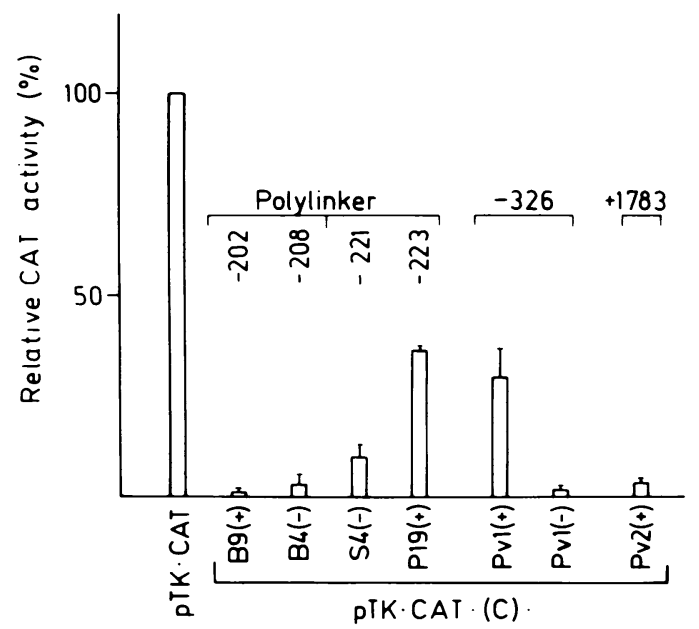

FIG. 5. Inhibition of TK promoter activity by fragment $C$ carrying the negative CMP control element. The numbers above the graph indicate the positions where fragment $C$ was inserted into the pTK . CAT vector $(+1$ corresponds to the translation initiation site of the TK gene). In the names of the constructs, insertion sites are denoted as follows: B, BamHI; P, PstI; Pv, PvuII; S, SalI. The ends of fragment $\mathrm{C}$ and the $S a l \mathrm{I}$ and PstI ends were treated with T4 DNA polymerase. BamHI ends were polished with nuclease S1. Clones were verified by sequence analysis. In B4(-), 3 nucleotides were removed $3^{\prime}$ of the BamHI cleavage site, while in $\mathrm{B} 9(+), 6$ and 9 nucleotides were removed from the vectors upstream and downstream, respectively. Results of four independent transfection experiments in chondrocytes using pTK . CAT control are shown. enhancer in the first intron is also characteristic for the type II collagen and other procollagen genes (12; for a review, see reference 3 ). The silencer fragment located immediately upstream of the TATA signal inhibited the promoter activity in both chondrocytes and fibroblasts. This finding suggests that the negative factors which bind to the silencer are also present in chondrocytes of 14-day-old chicken embryos. Similarly to the CMP silencer, the $\varepsilon$-globin silencer also inhibits its own promoter at the natural position both in erythroid and nonerythroid cell lines (4).

When the CMP enhancer was inserted upstream of the 1.2-kb CMP promoter fragment, it was not able to override the inhibition caused by the silencer region unless fragment B was deleted (Fig. 5). One explanation could be that additional, more remote enhancer sequences are also involved in transcriptional control of the gene. Alternatively, disruption of the natural organization of the regulatory elements in the constructs may alter the interactions of these elements with the promoter and with one another, resulting in a low level of gene expression. It has been reported that regulation of human $\alpha 1$ (I) collagen gene is highly influenced by the geometry of the control elements around the transcriptional start site via protein-mediated promoter-intron interactions (2).

It is also possible that the CMP promoter or enhancer or both display the highest activity when the gene is turned on in the prechondrogenic limb mesenchymal cells $(1,24)$, since the biosynthesis of CMP seems to be suppressed later during differentiation (6). A correlation between chondrogenesis and the enhancer activity of the rat type II collagen gene was published recently (12).

The experiments described in this paper provide a basis for the further characterization of the CMP regulatory elements and can promote identification of transacting factors involved in the regulation of cartilage-specific genes.

We are particularly grateful to A. Venetianer for the possibility of collaboration and stimulating discussions during this work. We thank C. Hadházy for technical advice on culturing chondrocyte and fibroblast cells; A. Simon and M. Lévai for excellent technical assistance; J. Varga and A. Borka for the artwork; and E. Fejes, J. Zákány, J. Gyuris, and I. Boros for critical reading of the manuscript. 
This work was supported by grant OKKFT Tt 1986/296 from the National Foundation of Technical Development of Hungary and grant OTKA 168 to I.K.

\section{LITERATURE CITED}

1. Argraves, W. S., F. Deák, K. J. Sparks, I. Kiss, and P. F. Goetinck. 1987. Structural features of cartilage matrix protein deduced from cDNA. Proc. Natl. Acad. Sci. USA 84:464-468.

2. Bornstein, P., J. McKay, D. J. Liska, S. Apone, and S. Devarayalu. 1988. Interactions between the promoter and first intron are involved in transcriptional control of $\alpha 1$ (I) collagen gene expression. Mol. Cell. Biol. 8:4851-4857.

3. Bornstein, P., and H. Sage. 1989. Regulation of collagen gene expression. Prog. Nucleic Acid Res. Mol. Biol. 37:67-106.

4. Cao, S. X., P. D. Gutman, H. P. G. Dave, and A. N. Schechter. 1989. Identification of a transcriptional silencer in the 5 '-flanking region of the human $\varepsilon$-globin gene. Proc. Natl. Acad. Sci. USA 86:5306-5309.

5. Deschatrette, J., and M. C. Weiss. 1974. Characterization of differentiated and dedifferentiated clones from a rat hepatoma. Biochimie 56:1603-1611.

6. Franzen, A., D. Heinegard, and M. Solursh. 1987. Evidence for sequential appearance of cartilage matrix proteins in developing mouse limbs and in cultures of mouse mesenchymal cells. Differentiation 36:199-210.

7. Goodbourn, S., H. Burstein, and T. Maniatis. 1986. The human $\beta$-interferon gene enhancer is under negative control. Cell 45:601-610.

8. Gorman, C. M., G. T. Merlino, M. C. Willingham, I. Pastan, and B. H. Howard. 1982. The Rous sarcoma virus long terminal repeat is a strong promoter when introduced into a variety of eukaryotic cells by DNA-mediated transfection. Proc. Natl. Acad. Sci. USA 79:6777-6781.

9. Gorman, C. M., L. F. Moffat, and B. H. Howard. 1982. Recombinant genomes which express chloramphenicol acetyltransferase in mammalian cells. Mol. Cell. Biol. 2:1044-1051.

10. Graham, F. L., and A. J. van der Eb. 1973. A new technique for the assay of infectivity of human adenovirus 5 DNA. Virology 52:456-467.

11. Hall, B. K. (ed.) 1983. Cartilage, vol. 3. Academic Press, Inc., New York.

12. Horton, W., T. Miyashita, K. Kohno, J. R. Hassel, and Y. Yamada. 1987. Identification of a phenotype-specific enhancer in the first intron of the rat collagen II gene. Proc. Natl. Acad. Sci. USA 84:8864-8868.

13. Kadesch, T., P. Zervos, and D. Ruezinsky. 1986. Functional analysis of the murine IgH enhancer: evidence for negative control of cell-type-specificity. Nucleic Acids Res. 14:8209
8221.

14. Khoury, G., and P. Gruss. 1983. Enhancer elements. Cell 33:313-314

15. Kishimoto, T. K., R. S. Larson, A. L. Corbi, M. L. Dustin, D. E. Staunton, and T. A. Springer. 1989. The leukocyte integrins. Adv. Immunol. 46:149-182.

16. Kiss, I., F. Deák, R. G. Holloway, Jr., H. Delius, K. A. Mebust, E. Frimberger, W. S. Argraves, P. A. Tsonis, N. Winterbottom, and P. F. Goetinck. 1989. Structure of the gene for cartilage matrix protein, a modular protein of the extracellular matrix. J. Biol. Chem. 264:8126-8134.

17. Kiss, I., F. Deák, S. Mestric, H. Delius, J. Soós, K. Dékány, W. S. Argraves, K. J. Sparks, and P. F. Goetinck. 1987. Structure of the chicken link protein gene: exons correlate with the protein domains. Proc. Natl. Acad. Sci. USA 84:6399-6403.

18. Kosher, R. A., S. W. Gay, J. R. Kamanitz, W. M. Kulyk, B. J. Rodgers, S. Sai, T. Tanaka, and M. L. Tanzer. 1986. Cartilage proteoglycan core protein gene expression during limb cartilage differentiation. Dev. Biol. 118:112-117.

19. Lovell-Badge, R. H., A. Bygrave, A. Bradley, E. Robertson, R. Tilly, and K. S. E. Cheah. 1987. Tissue-specific expression of the human type II collagen gene in mice. Proc. Natl. Acad. Sci. USA 84:2803-2807.

20. Luciw, P. A., J. M. Bishop, H. E. Varmus, and M. R. Capecchi. 1983. Location and function of retroviral and SV40 sequences that enhance biochemical transformation after microinjection of DNA. Cell 33:705-715.

21. Miksicek, R., A. Heber, W. Schmid, U. Danesch, G. Posseckert, M. Beato, and G. Schütz. 1986. Glucocorticoid responsiveness of the transcriptional enhancers of Moloney murine sarcoma virus. Cell 46:283-290.

22. Paulsson, M., and D. Heinegard. 1979. Matrix proteins bound to associatively prepared proteoglycans from bovine cartilage. Biochem. J. 183:539-545.

23. Pytela, R. 1988. Amino acid sequence of the murine Mac-1 a chain reveals homology with the integrin family and an additional domain related to von Willebrand factor. EMBO J. 7:1371-1378

24. Stirpe, N. S., and P. F. Goetinck. 1989. Gene regulation during cartilage differentiation: temporal and spatial expression of link protein and cartilage matrix protein in the developing limb. Development 107:23-34.

25. Venetianer, A., Z. Pintér, and A. Gál. 1980. Examination of glucocorticoid sensitivity and receptor content of hepatoma cell lines. Cytogenet. Cell Genet. 28:280-283.

26. Wasylyk, C., and B. Wasylyk. 1986. The immunoglobulin heavychain B-lymphocyte enhancer efficiently stimulates transcription in nonlymphoid cells. EMBO J. 5:553-560. 